\title{
Power Control in Cognitive Radio Networks Using Cooperative Modulation and Coding Classification
}

\author{
Anestis Tsakmalis, Symeon Chatzinotas and Björn Ottersten \\ SnT - securityandtrust.lu, University of Luxembourg \\ Email:\{anestis.tsakmalis, symeon.chatzinotas, bjorn.ottersten\}@uni.lu
}

\begin{abstract}
In this paper, a centralized Power Control (PC) scheme aided by interference channel gain learning is proposed to allow a Cognitive Radio (CR) network to access the frequency band of a Primary User (PU) operating based on an Adaptive Coding and Modulation (ACM) protocol. The main idea is the CR network to constantly probe the band of the PU with intelligently designed aggregated interference and sense whether the Modulation and Coding scheme (MCS) of the PU changes in order to learn the interference channel gains. The coordinated probing is engineered by the Cognitive Base Station (CBS), which assigns appropriate $C R$ power levels in a binary search way. Subsequently, each CR applies a Modulation and Coding Classification (MCC) technique and sends the sensing information through a control channel to the CBS, where all the MCC information is combined using a fusion rule to acquire an MCS estimate of higher accuracy and monitor the probing impact to the PU MCS. After learning the normalized interference channel gains towards the $P U$, the CBS selects the CR power levels to maximize total $C R$ network throughput while preserving the PU MCS and thus its QoS. The effectiveness of the proposed technique is demonstrated through numerical simulations.
\end{abstract}

Keywords-Cognitive Radio, Centralized Power Control, Spectrum Sensing, Cooperative Modulation and Coding Classification, Adaptive Coding and Modulation

\section{INTRODUCTION}

Radio Spectrum is well known to be a limited resource. Ever since its first commercial usage, regulations for limiting services to specific frequency bands have been enforced. This rulemaking process assumes that a static assignment of services to frequency bands not only facilitates the financial exploitation of the Radio Spectrum, but also limits interference and supports the construction of cheap and less complicated transceivers, a major technological restraint.

Nowadays though, the burst in service demand has led us to rethink the static nature of this architecture. Taking into account also the fact that some frequency bands are being underutilized and that others accommodate services resilient to interference, the research community proposed the idea of the Dynamic Spectrum Access (DSA) [1]. Some DSA techniques suggest the use of frequency bands by unlicensed users, also called Secondary Users (SUs), when the licensed ones (PUs) are absent or even their coexistence as long as the received interference by PUs is below a certain threshold. This flexible structure enables us to exploit the Radio Spectrum resource more efficiently. A candidate technology to reach this objective and enhance the operation of the SUs is the
Cognitive Radio (CR) [2]. The main functionalities of the CR are the Spectrum Sensing (SS), which consists of methods detecting the existence or type of licensed primary signal, and the PC, the adaptive adjustment of transmit power. Unlicensed SUs equipped with these CR mechanisms can apply DSA techniques and help us resolve both Radio Spectrum underutilization and congestion.

One important SS mechanism is the identification of the PU signal type. An interesting approach to address this problem could be the classification of the modulation and coding scheme (MCC) [3], [4]. As far as the modulation classification is concerned, features like the signal cumulants of $2 \mathrm{nd}$, 3rd, 4th, 6th and 8th order which have distinctive theoretical values among different modulation schemes [5] are estimated and then fed into a powerful classification tool, the Support Vector Machine (SVM) [6]. For the coding identification part, the exploited statistical features are the log-likelihood ratios (LLRs) of the received symbol samples [7], [8]. The detection technique in this case involves the comparison of the average LLRs of the error syndromes derived from the parity-check relations of each code.

The second CR enhancement mentioned before is the PC strategy based on which the SUs are accessing the frequency band of the PU. This vast topic has been thoroughly investigated from many aspects depending on the system model, the optimization variables, the objective functions, the constraints and other known or unknown parameters. An interesting approach to the PC problem tackled by the research community within the wireless network context has been the centralized one. Based on this, a central decision maker, the Base Station, gathers local information from the users through a control channel, elaborates an intelligent selection of their operational parameters, such as their transmit power, channel or time schedule, and communicates it to them. In this general context, the research community has formulated and tackled PC problems [9-14] to achieve common or different signal to interference plus noise ratio $(S I N R)$ requirements, maximum total system throughput, maximum weighted throughput, maximum worst user throughput or minimum transmit power, subject to QoS constraints from individual users, like $S I N R$, data rate or outage probability. In the CR regime, the centralized PC problem retains its basic form but with some small alterations. One critical modification is the knowledge of the interference channels from the CR transmitters to the PU receivers. Previous work has considered perfect CR-toPU channel knowledge [15], [16], limited-rate feedback from 
the PUs on CR-to-PU channel gains [17], imperfect CR-to-PU channel knowledge [18] and CR-to-PU channel uncertainty knowledge attained through SS or channel gain cartography [19].

An even more challenging PC problem in CR networks is the one without any prior knowledge of the interference channels and cooperation from the PU link. The additional burden in this case is learning the CR-to-PU channels using eavesdropped information from the PU feedback channel. A solution for one SU coexisting with one PU was given in [20] based on a probing and sensing model. Nevertheless, the most sophisticated methods suitable for learning the interference channel gains of multiple SUs through probing with the use of even binary feedback are derived from multiple input multiple output (MIMO) and beamforming research scenarios. Previous researchers have exploited a slow random exploration algorithm [21], the one-bit null space learning algorithm [22] and an analytic center cutting plane method (ACCPM) based learning algorithm [23].

In this paper, a centralized PC method aided by interference channel power gain learning is demonstrated which concerns multiple SUs and a PU and maximizes the total SU throughput subject to maintaining the PU QoS. This case study considers the PU link changing its MCS based on an ACM protocol and operating in its assigned band together with a CR network accessing this band and having knowledge of this ACM protocol. Our idea is to apply an algorithm in order to first estimate the interference channel power gains by exploiting SS feedback and finally maximize the total SU throughput. This CR-to-PU channel knowledge is acquired by having the coexisting cognitive SUs constantly probing in a binary search trial and error way and checking whether the CR network caused the PU MCS to change. The detection of the PU MCS is performed in a cooperative way at the CBS which gathers the MCC feedback from all the SUs through a control channel and combines them using a hard decision fusion rule. The proposed DSA application concerns only the SU system without adding any complexity in the infrastructure or a control channel between the PU system and the SU one in order to exchange information about the channel gains or the induced interference.

The remainder of this paper is structured as follows: Section II provides the system model and the problem formulation. Section III introduces the cooperative MCC. Section IV analyzes the interference channel power gain learning. Section V shows the results obtained by the combination of the above. Finally, Section VI gives the concluding remarks and future work in this topic.

\section{System Model AND PROBlem Formulation}

Initially, the outputs of the MCC procedure and the way they are employed have to be described. All the SUs are equipped with a secondary omnidirectional antenna only for sensing the PU signal and an MCC module which enables them to identify the MCS of the PU as shown in Fig. 1. Specifically, each SU collects PU signal samples using a standard sensing period $T_{S}$, estimates the current MCS and transmits it through a control channel to the CBS. The MCS observation of the $\mathrm{SU}_{\mathrm{i}}$ over the $\mathrm{n}_{\text {th }}$ sensing period is expressed as $M C S_{i}^{n}$ and a detailed description about its estimation can be found in [3], [4].

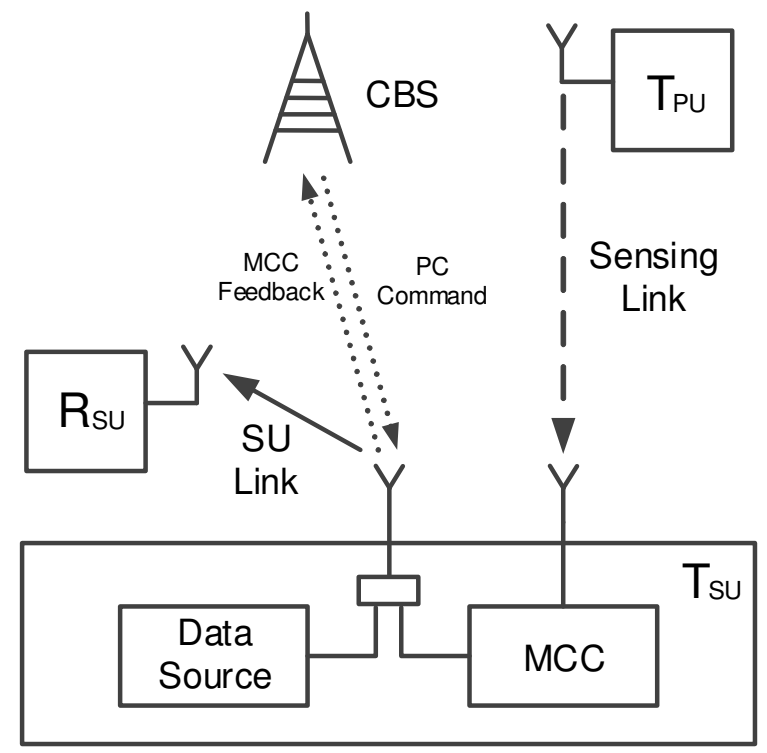

Fig. 1. Abstract illustration of the CR operation

Furthermore, at system level a PU link and $N$ SU links exist in the same frequency band as shown in Fig. 2. As far as the interference to the PU link is concerned, this is caused by the transmitter part of each SU link to the receiver of the PU link. Considering strong interference links, this may have a severe effect on the MCS chosen by the PU link. In addition, a channel access method allows SU links not to interfere with each other. In this scenario, the unknown interference channel gains and the PU channel gain are static and no fading channel models are considered.

Here we focus on channel power gains $G$, which in general are defined as $G=\|g\|^{2}$, where $g$ is the channel gain. From this point, we will refer to channel power gains as channel gains. The aggregated interference to the PU side is defined as:

$$
I_{P U}=\sum_{i=1}^{N} G_{I_{i}} P_{S U_{i}}
$$

where $G_{I_{i}}$ is the $\mathrm{SU}_{\mathrm{i}}$-to-PU interference channel gain and $P_{S U_{i}}$ is the $\mathrm{SU}_{\mathrm{i}}$ transmit power. Additionally, the $S I N R$ of the PU is defined in as:

$$
S I N R_{P U}=10 \log \left(\frac{G_{P U} P_{P U}}{I_{P U}+N_{P U}}\right) \mathrm{dB}
$$

where $G_{P U}$ is the PU link channel gain, $P_{P U}$ is the PU transmit power and $N_{P U}$ is PU receiver noise power. From a PU system perspective, an ACM scheme is applied with a set of possible MCS's. The ACM protocol changes the MCS of the PU link to more or less robust modulation constellations and coding rates depending on the level of the $S I N R_{P U}$. Each MCS operation has a specific minimum required $S I N R_{P U}$ 


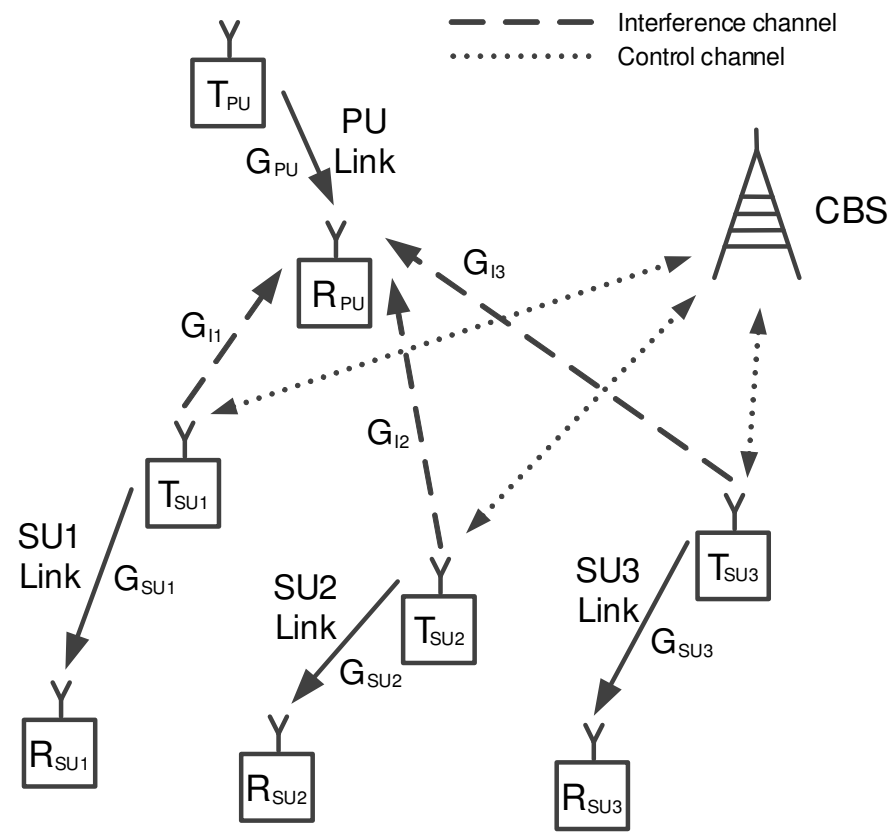

Fig. 2. The PU system and the CR network

value, denoted as $S I N R_{t h}$, which whenever violated, an MCS adaptation happens. Assuming that $N_{P U}$ remains the same at the PU receiver side and that the PU transmitter retains its transmit power, the $S I N R_{t h}$ values correspond to particular maximum allowed $I_{P U}$ values, designated as $I_{t h}$. Hence, whenever the PU is active, for every MCS there are interference thresholds $I_{t h}$ over which the PU is obliged to change its transmission scheme to a lower order modulation constellation or a lower code rate and whose levels are unknown to the CR network.

In this paper, we address the problem of total SU throughput $\left(U_{S U}^{t o t}\right)$ maximization without causing harmful interference to the PU system, which can be written as:

$$
\begin{array}{ll}
\underset{\mathbf{P}_{\mathrm{SU}}}{\operatorname{maximize}} & U_{S U}^{t o t}\left(\mathbf{P}_{\mathrm{SU}}\right)=\sum_{i=1}^{N} \log _{2}\left(1+\frac{G_{S U_{i}} P_{S U_{i}}}{N_{S U_{i}}}\right) \\
\text { subject to } & \sum_{i=1}^{N} G_{I_{i}} P_{S U_{i}} \leq I_{t h} \\
& 0 \leq P_{S U_{i}} \leq P_{i}^{\max } i=1, \ldots, N
\end{array}
$$

where $\mathbf{P}_{\mathbf{S U}}$ is the power vector $\left[P_{S U_{1}}, \ldots, P_{S U_{N}}\right], P_{i}^{\max }$ is the maximum transmit power level of the $\mathrm{SU}_{\mathrm{i}}$ transmitter, $G_{S U_{i}}$ is the channel gain of the $\mathrm{SU}_{\mathrm{i}}$ link and $N_{S U_{i}}$ is the noise power level of the $\mathrm{SU}_{\mathrm{i}}$ receiver. The channel gain parameters $G_{S U_{i}}$ and the noise power levels $N_{S U_{i}}$ are considered to be known to the CR network and not changing in time. An observation necessary for tackling this problem is that the $G_{I_{i}}$ gains normalized to $I_{t h}$ are adequate for defining the interference constraint. Therefore, the new version of ( $3 b)$, will be:

$$
\sum_{i=1}^{N} G_{I_{i}}^{n o r m} P_{S U_{i}} \leq 1
$$

where $G_{I_{i}}^{\text {norm }}=\frac{G_{I_{i}}}{I_{t h}}$.

This optimization problem is convex and using the KarushKuhn-Tucker (KKT) approach a capped multilevel waterfilling (CMP) solution is obtained [24] for each $\mathrm{SU}_{\mathrm{i}}$ of the closed form:

$$
P_{S U_{i}}^{*}=\left\{\begin{array}{cc}
P_{i}^{\max } & \text { if } \frac{1}{\lambda G_{I_{i}}^{\text {norm }}}-\frac{N_{S U_{i}}}{G_{S U_{i}}} \geq P_{i}^{\max } \\
0 & \text { if } \frac{1}{\lambda G_{I_{i}}^{\text {orm }}}-\frac{N_{S U_{i}}}{G_{S U_{i}}} \leq 0 \\
\frac{1}{\lambda G_{I_{i}}^{\text {norm }}-\frac{N_{S U_{i}}}{G_{S U_{i}}}} & \text { otherwise }
\end{array}\right.
$$

where $\lambda$ is the KKT multiplier of the interference constraint (4) and which can be determined as presented in [24]. Once, all the parameters of the optimization problem are established, the aforementioned analytical solution can be directly calculated. In the following sections, we deal with the learning of the unknown parameters described in the constraint (4).

\section{Cooperative Modulation and Coding CLASSIFICATION}

A general description of cooperative SS is that each SU performs a SS technique independently, forwards its observation to the CBS via a control channel and finally the CBS using a fusion rule combines this information to get to a decision. In this paper, a hard decision fusion of observations obtained by MCC is considered using a plurality voting system [25]. Based on this voting system, the CBS collects all the MCC feedback over the $n_{t h}$ sensing period and decides the MCS of the PU, denoted as $M C S^{n}$. Let $C=\left\{c_{1}, . ., c_{K}\right\}$ denote the set of the MCS candidates of the ACM protocol, which are considered to be equiprobable, $K$ the number of elements of this set and $V_{c_{j}}$ the vote tally associated with the class $c_{j}$.

During the voting procedure, the CBS first gathers the votes of the $n_{t h}$ period, which in our case are the $M C S_{1}^{n}, \ldots, M C S_{N}^{n}$ and support elements of the class set $C$. All the votes are of same importance and no use of weight factors is made. With every vote $M C S_{i}^{n}$, the CBS increases by one the vote tally $V_{c_{j}}$ of the $c_{j}$ class supported by this vote. After casting every vote of the $n_{t h}$ period to the corresponding vote tally, the CBS identifies the $M C S^{n}$ as:

$$
M C S^{n}=\arg \max _{c_{j} \in C} V_{c_{j}} .
$$

Even though plurality voting is a simple and not sophisticated method which elects the MCS value that appears more often than all of the others, it produces the correct voting output under the condition that some SUs have sensing channels of moderate quality. Its equivalent voting system for binary data fusion, the majority one, has been used by the research community to improve the detection and false alarm probabilities with satisfactory results. 


\section{INTERFERENCE HYPERPLANE LEARNING}

From here on, the equality extreme of the constraint (4) will be referred to as the interference hyperplane. In this section, a binary search probing method is described for estimating the interference hyperplane. First of all, a binary indicator is defined which shows whether the CR network is generating $I_{P U}$ above or below the $I_{t h}$ based on the $M C S^{n}$ fusion output of the $M C S_{i}^{n}$ observations. Whenever the CBS detects a deterioration of the MCS from the $(n-1)_{t h}$ to the $n_{t h}$ period, the indicator $I^{n}$ changes state as shown below:

$$
I^{n}=\left\{\begin{array}{ll}
1 & \text { if } M C S^{n} \neq M C S^{n-1} \\
0 & \text { if } M C S^{n}=M C S^{n-1}
\end{array} .\right.
$$

In addition, the feasible set of this problem is defined as $\Omega^{N}=\left\{\mathbf{P}_{\mathrm{SU}} \mid 0 \leq P_{S U_{i}} \leq P_{i}^{\max }, i=1, \ldots, N\right\}$, an $N-$ dimensional rectangle with $2^{N}$ corners. The objective of this section is to find a geometric method for determining this hyperplane which crosses $\Omega^{N}$. The only exploitable feedback of this method is the $I^{n}$ which specifies whether the SU power allocation $\mathbf{P}_{\mathbf{S U}}$, chosen by the CBS, just before the beginning of the $n_{t h}$ period causes or not harmful interference. In other words, if the SU power allocation before the beginning of the $n_{t h}$ period is expressed as $\mathbf{P}_{\mathbf{S U}}^{\mathbf{n}}=\left[P_{S U_{1}}^{n}, \ldots, P_{S U_{N}}^{n}\right], I^{n}$ demonstrates whether this chosen point in $\Omega^{N}$ is above or below the interference hyperplane.

Thus, the main challenge of this method is how to intelligently select a series of points in $\Omega^{N}$, for which we only know whether they are above or below the desired hyperplane, in order to estimate this hyperplane. Also, this series has to be limited within the $N$-dimensional rectangle, since the CBS cannot assign power levels beyond this region. Another challenge that has to be taken into account is the total number of these probing/testing points. The more trial points are used, the more MCS deteriorations the CR network likely causes, which is a considerable damage to the PU QoS. So, this geometric method must find the interference hyperplane with the lowest number of trial points possible.

The core idea for solving the problem is the limitation of the feedback $I^{n}$. A binary indicator would be ideal to determine a threshold in the 1-dimensional case by using binary search. Still, in the $N$-dimensional case a binary searchlike method must have some kind of directivity to identify the hyperplane-threshold. Hence, the question becomes how can binary search be applied in this scenario. Basically, to detect an $N$-dimensional plane one has to find $N$ linearly independent points upon it. Furthermore, if each point belongs to a 1-dimensional ordered set, like a line segment, the binary indicator $I^{n}$ could be used for a binary search upon the set to find this point. Consequently, for this idea to work, $N$ line segments which cross the hyperplane need to be found and with the lowest number of trial points possible.

To locate $N$ line segments crossing the hyperplane, a number of end points need to be known with some of them below the $N$-dimensional plane and the rest above it. Considering that any combination of points from different sides creates line segments which cross the hyperplane, if points above and below the $N$-dimensional plane belong respectively to groups $\mathrm{A}$ and $\mathrm{B}$ and $N_{A}$ and $N_{B}$ are the number of points in these groups, then the number of possible line segments is $N_{A} N_{B}$. As mentioned before, the required number of line segments is $N$, but since the lowest number of trial points possible is demanded the problem is to find $N_{A}$ and $N_{B}$ points minimizing $N_{A}+N_{B}$ while $N_{A} N_{B} \geq N$.

Taking into account some facts from the nature of this problem, the aforementioned end point search can be simplified. Given that the interference hyperplane crosses $\Omega^{N}$, there is always a known point below this $N$-dimensional plane, the $[0, \ldots, 0]$, and one above it, the $\left[P_{1}^{\max }, \ldots, P_{N}^{\max }\right]$. So, in the worst case scenario, $N-1$ more points are needed to define $N$ line segments crossing the hyperplane. To simplify the end point search, it is proposed to examine randomly the corners of the $\Omega^{N}$. After these segments are found, binary searches are performed on each one of them so as to detect the $N$ intersection points of the line segments and hence the interference hyperplane.

A detailed description of the binary search method on a line segment with arbitrary end points should also be given. Assuming 2 points, $\mathbf{p}_{\mathbf{1}}$ and $\mathbf{p}_{\mathbf{2}}$, in the $N$-dimensional space, every point $\mathbf{p}(\theta)$ lying on the line segment defined by them is expressed using the parametric equation $\mathbf{p}(\theta)=\theta \mathbf{p}_{\mathbf{1}}+$ $(1-\theta) \mathbf{p}_{\mathbf{2}}$, where $\theta \in[0,1]$. So, basically the binary search is performed within the $\theta$ region $[0,1]$.

Once, the intersection points of the line segments and the interference hyperplane are estimated the $G_{I_{i}}$ gains normalized to $I_{t h}$ can be found as the solution of an $N \times N$ system using the equality of the constraint (4):

$$
\left[\begin{array}{c}
G_{I_{1}}^{\text {norm }} \\
G_{I_{2}}^{\text {norm }} \\
\vdots \\
G_{I_{N}}^{\text {norm }}
\end{array}\right]=\left[\begin{array}{c}
\mathbf{P}_{1}^{\text {cross }} \\
\mathbf{P}_{2}^{\text {cross }} \\
\vdots \\
\mathbf{P}_{\mathbf{N}}^{\text {cross }}
\end{array}\right]^{-1}\left[\begin{array}{c}
1 \\
1 \\
\vdots \\
1
\end{array}\right]
$$

where $\mathbf{P}_{\mathbf{i}}^{\text {cross }}, i=1, \ldots, N$, are the intersection points as row vectors.

Additionally, it is necessary to determine the maximum probing/testing points needed to detect the intersection points and thus the interference hyperplane, since it was explained that a large number of probing/testing points could degrade the PU QoS. Supposing that each binary search is performed with accuracy $\epsilon$, it is well known that the maximum attempts for each line segment of length $d_{k}$, where $k=1, \ldots, N$, are $\left\lceil\log _{2}\left(\frac{d_{k}}{\epsilon}\right)\right\rceil$. Even though the lengths $d_{k}$ cannot be precisely estimated, because a random selection of corner points is performed and so the line segments do not have a standard length, an upper bound can be derived for the total binary search attempts of the procedure. The maximum length a line segment can have in $\Omega^{N}, d_{\max }$, is of the diagonal defined by the points $[0, \ldots, 0]$ and $\left[P_{1}^{\max }, \ldots, P_{N}^{\max }\right]$ and calculated as

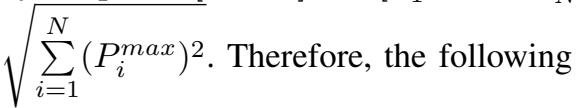

$$
\sum_{k=1}^{N}\left\lceil\log _{2}\left(\frac{d_{k}}{\epsilon}\right)\right\rceil \leq N\left\lceil\log _{2}\left(\frac{d_{\max }}{\epsilon}\right)\right\rceil
$$




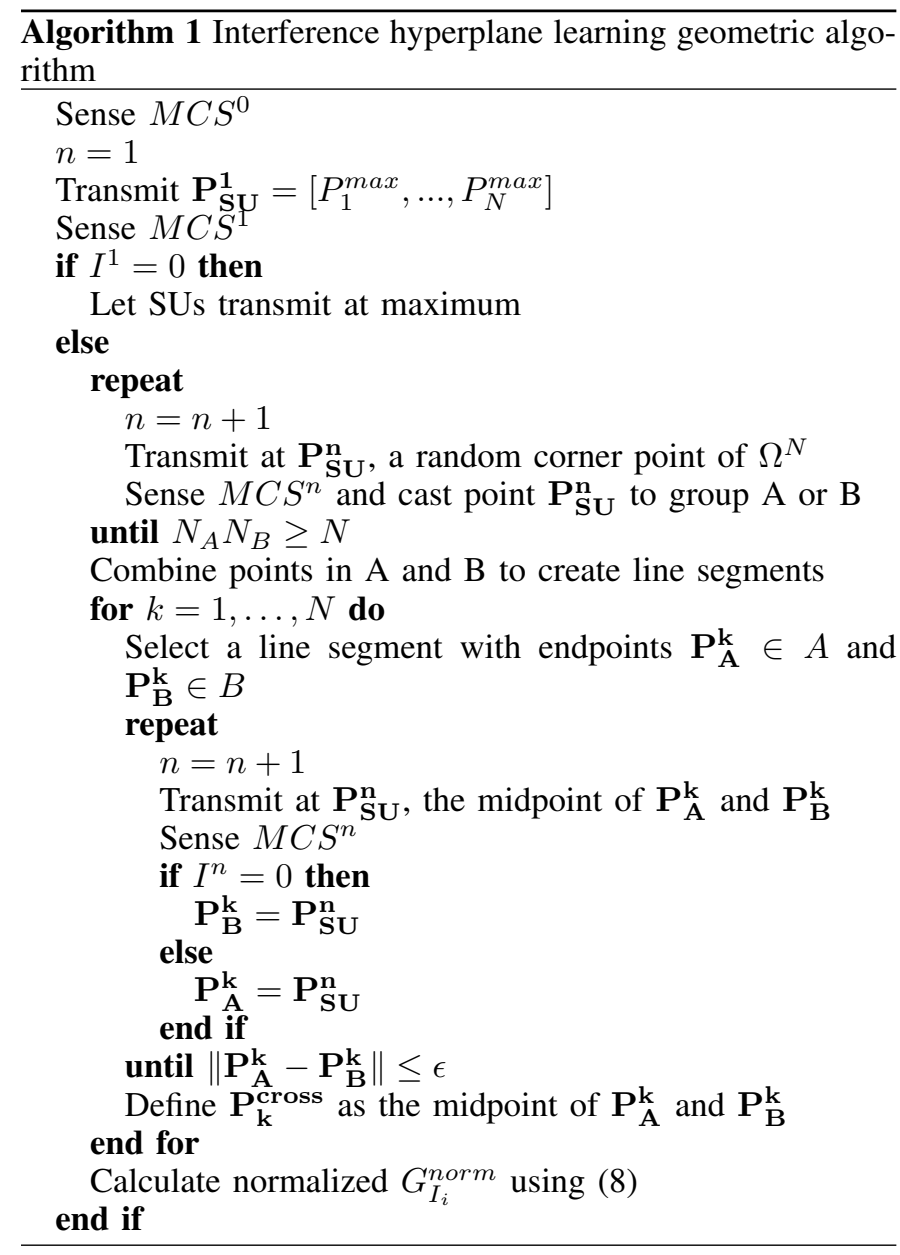

holds and presents an upper boundary of $\mathcal{O}\left(N \log _{2}(N)\right)$ performing trials. This result proves the scalability of this geometric algorithm, presented in Algo. 1, which can be used even when the SUs of the CR network are large in number.

A simple example of how this geometric algorithm progresses in time for $N=2$ SUs is given in Fig. 3. The binary searches were performed on the line segments $O B$ and $B C$ in order to find their intersection points with the interference line, $E$ and $D$. Once, these points are obtained it is easy to define the interference line.

\section{RESULTS}

Following, the performance of the aforementioned geometric algorithm and the probing progress of each $P_{S U_{i}}$ vs time are presented. For testing the performance, a case of CR network with $N=3$ SUs was considered. The following diagrams in Fig. 4 represent geometrically the probing/testing point coordinates which gradually converge to the coordinates of the intersection points $\mathbf{P}_{\mathbf{i}}^{\text {cross }}$.

As seen in Fig. 4, testing power allocation points are tried with a time step of $200 \mathrm{~ms}$ and it is considered that $P^{\max }=300 \mathrm{~mW}$ and $T_{S}=100 \mathrm{~ms}$ for every SU. Also, at each time step the output of the cooperative MCC process is

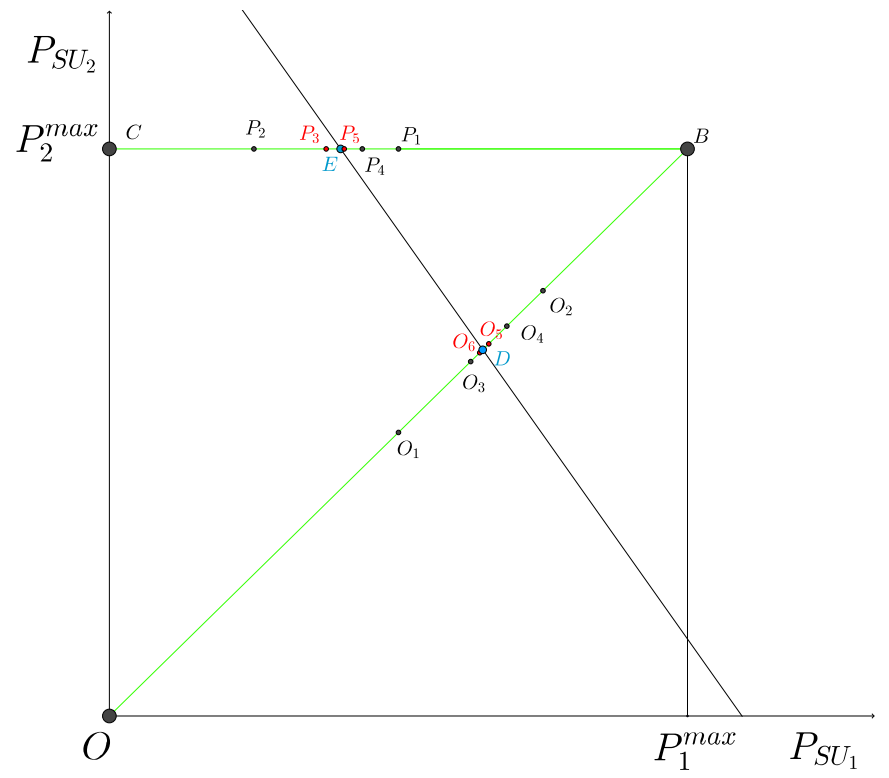

Fig. 3. A 2D graphical example of the geometric algorithm
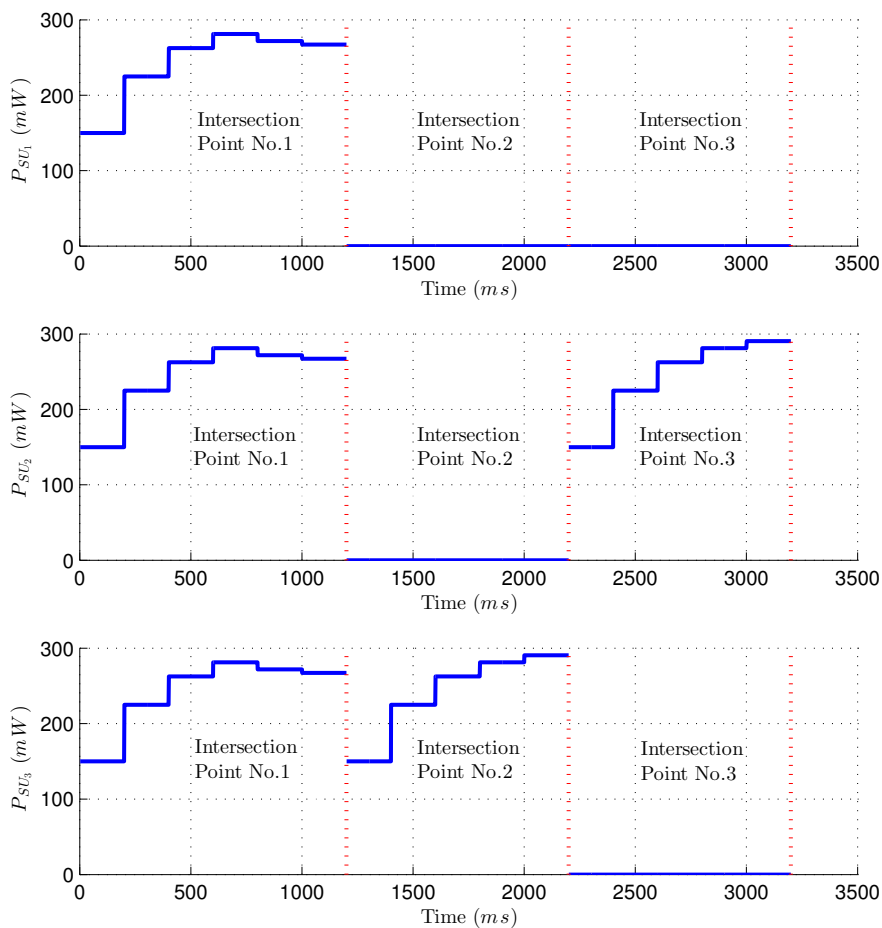

Fig. 4. Geometric algorithm progress in time for $N=3$ SUs

assumed to be correctly estimated and thus the binary indicator $I^{n}$ contributing in the geometric algorithm is always accurate. An important aspect of the algorithm is the convergence time which for the examined CR network is $3200 \mathrm{~ms}$. After the interference hyperplane learning process finishes, the CBS is able to directly find the optimal power allocation based on (5). 


\section{CONCLUSIONS}

In this paper, a centralized Power Control (PC) scheme aided by an interference hyperplane learning algorithm is proposed to allow a CR network and a PU coexist in a frequency band. The leading idea of this algorithm is to exploit the SS feedback from the cooperative MCC procedure and perform consecutive binary searches in the power allocation set to define points of the interference hyperplane and thus the hyperplane itself. The algorithm is of $\mathcal{O}\left(\mathrm{Nlog}_{2}(N)\right)$ time complexity, which makes it ideal even for large CR networks, and guarantees that the minimum number of probing/testing points possible is performed. The last remark is essential for the PU QoS, since the more trials are performed by the CBS for the hyperplane learning, the more likely it is to surpass the interference hyperplane and deteriorate the PU MCS. Moreover, it must be mentioned that the proposed learning algorithm achieves better time complexity than the ones used in previous work related with binary feedback learning. In [21], a slow convergence rate stochastic gradient algorithm was utilized, [22] suggested an algorithm of $\mathcal{O}\left(N^{2} \log _{2}(N)\right)$ time complexity and [23] applied an optimization technique based learning algorithm of $\mathcal{O}\left(N^{2}\right)$ time complexity.

Improving some of the problem aspects could lead our future work in this subject. Initially, an enhanced fusion rule of the MCC observations could be suggested using soft decision rules based on the sensing link quality of each SU. Furthermore, the cooperative MCC process is assumed to perfectly recognize the PU MCS, but under low quality sensing link conditions this is not true. An introduction of a reliability factor indicating how accurate the output of the MCS fusion rule is and therefore how reliable the binary indicator $I^{n}$ is could be useful so that binary searches using uncertainty could be carried out. Finally, an online version of the proposed geometric algorithm could be implemented to tackle fading interference channels and not only static ones.

\section{ACKNOWLEDGEMENT}

This work was supported by the National Research Fund, Luxembourg under the CORE project "SeMIGod: SpEctrum Management and Interference mitiGation in cOgnitive raDio satellite networks".

\section{REFERENCES}

[1] Q. Zhao and B. Sadler, "A Survey of Dynamic Spectrum Access," IEEE Signal Processing Magazine, pp. 79-89, 2007.

[2] J. Mitola, "Cognitive radio an integrated agent architecture for software defined radio," Ph.D. dissertation, KTH Royal Institute of Technology Stockholm, Stockholm, Sweden, 2000.

[3] A. Tsakmalis, S. Chatzinotas, and B. Ottersten, "Modulation and Coding Classification for Adaptive Power Control in 5G Cognitive Communications," IEEE International Workshop on Signal Processing Advances in Wireless Communications (SPAWC), 2014.

[4] A. Tsakmalis, S. Chatzinotas, and B. Ottersten, "Automatic Modulation Classification for Adaptive Power Control in Cognitive Satellite Communications," 7th Advanced Satellite Multimedia Systems Conference (ASMS) and 13th Signal Processing for Space Communications Workshop (SPSC), 2014.
[5] B. Ramkumar, "Automatic Modulation Classification for Cognitive Radios Using Cyclic Feature Detection," IEEE Circuits and Systems Magazine, pp. 27-45, 2009.

[6] V. N. Vapnik, The Nature of Statistical Learning Theory. Springer, 1999.

[7] T. Xia and H. Wu, "Novel Blind Identification of LDPC Codes Using Average LLR of Syndrome a Posteriori Probability," IEEE Transactions on Signal Processing, pp. 632-640, 2014.

[8] R. Moosavi and E. Larsson, "A Fast Scheme for Blind Identification of Channel Codes," IEEE Global Telecommunications Conference (GLOBECOM), pp. 1-5, 2011.

[9] R. Yates, "A Framework for Uplink Power Control in Cellular Radio Systems," IEEE Journal on Selected Areas in Communications, pp. 1341-1347, 1995.

[10] S. Grandhi, R. Vijayan, D. Goodman, and J. Zander, "Centralized Power Control in Cellular Radio Systems," IEEE Transactions on Vehicular Technology, pp. 466-468, 1993.

[11] L. Qian, Y. Zhang, and J. Huang, "MAPEL: Achieving Global Optimality for a Non-Convex Wireless Power Control Problem," IEEE Transactions on Wireless Communications, pp. 1553-1563, 2009.

[12] M. Chiang, C. W. Tan, D. Palomar, D. ONeill, and D. Julian, "Power Control By Geometric Programming," IEEE Transactions on Wireless Communications, pp. 2640-2651, 2007.

[13] C. W. Tan, D. Palomar, and M. Chiang, "Solving Nonconvex Power Control Problems in Wireless Networks: Low SIR Regime and Distributed Algorithms," IEEE Global Telecommunications Conference (GLOBECOM), 2005.

[14] S. Kandukuri and S. Boyd, "Optimal Power Control in InterferenceLimited Fading Wireless Channels With Outage-Probability Specifications," IEEE Transactions on Wireless Communications, pp. 46-55, 2002.

[15] I. Mitliagkas, N. Sidiropoulos, and A. Swami, "Convex ApproximationBased Joint Power and Admission Control for Cognitive Underlay Networks," International Wireless Communications and Mobile Computing Conference (IWCMC), pp. 28-32, 2008.

[16] R. Zhang and Y. C. Liang, "Exploiting Multi-Antennas for Opportunistic Spectrum Sharing in Cognitive Radio Networks," IEEE Journal of Selected Topics in Signal Processing, pp. 88-102, 2008.

[17] A. Marques, X. Wang, and G. Giannakis, "Dynamic Resource Management for Cognitive Radios Using Limited-Rate Feedback," IEEE Transactions on Signal Processing, pp. 3651-3666, 2009.

[18] I. Mitliagkas, N. Sidiropoulos, and A. Swami, "Joint Power and Admission Control for Ad-Hoc and Cognitive Underlay Networks: Convex Approximation and Distributed Implementation," IEEE Transactions on Wireless Communications, pp. 4110-4121, 2011.

[19] E. Dall'Anese, S. Kim, G. Giannakis, and S. Pupolin, "Power Control for Cognitive Radio Networks Under Channel Uncertainty," IEEE Transactions on Wireless Communications, pp. 3541-3551, 2011.

[20] I. Bajaj and Y. Gong, "Cross-Channel Estimation Using Supervised Probing and Sensing in Cognitive Radio Networks," IEEE International Conference on Communications (ICC), pp. 1-5, 2011.

[21] B. C. Banister and J. R. Zeidler, "A Simple Gradient Sign Algorithm for Transmit Antenna Weight Adaptation With Feedback," IEEE Transactions on Signal Processing, pp. 1156-1171, 2003.

[22] Y. Noam and A. J. Goldsmith, "The One-Bit Null Space Learning Algorithm and Its Convergence," IEEE Transactions on Signal Processing, pp. 6135-6149, 2013.

[23] J. Xu and R. Zhang, "Energy Beamforming With One-Bit Feedback," IEEE Transactions on Signal Processing, pp. 5370-5381, 2014.

[24] L. Zhang, Y. C. Liang, and Y. Xin, "Joint Beamforming and Power Allocation for Multiple Access Channels in Cognitive Radio Networks," IEEE Journal on Selected Areas in Communications, pp. 617-629, 2008.

[25] B. Parhami, "Voting Algorithms," IEEE Transactions on Reliability, pp. 617-629, 1994. 